\title{
An Efficient Numerical Algorithm For A Multiphase Tumour Model
}

\author{
Alrehaili $\mathrm{AH}^{\mathrm{a}, \mathrm{b}}$, Walkley $\mathrm{MA}^{\mathrm{a}}$, Jimack $\mathrm{PK}^{\mathrm{a}}$, Hubbard $\mathrm{ME}^{\mathrm{c}}$ \\ ${ }^{a}$ School of Computing, University of Leeds, Leeds, UK \\ ${ }^{b}$ School of Mathematics, Taibah University, Medina, Saudi Arabia \\ ${ }^{c}$ School of Mathematical Sciences, University of Nottingham, Nottingham, UK
}

\begin{abstract}
This paper is concerned with the development and application of optimally efficient numerical methods for the simulation of vascular tumour growth. This model used involves the flow and interaction of four different, but coupled, phases which are each treated as incompressible fluids, [1]. A finite volume scheme is used to approximate mass conservation, with conforming finite element schemes to approximate momentum conservation and an associated equation. The principal contribution of this paper is the development of a novel block preconditioner for solving the linear systems arising from the discrete momentum equations at each time step. In particular, the preconditioned system has both a solution time and a memory requirement that is shown to scale almost linearly with the problem size.
\end{abstract}

Keywords: Multiphase system, Tumour model, Numerical simulations, Preconditioning.

\section{Introduction}

In recent decades, research related to tumour growth has increased dramatically and mathematical tumour modelling has made a significant contribution to this. These mathematical models complement biological experiments and clinical observations, and simulations of tumours can help to support the hypotheses taken from such observations. The review paper [2]

Email addresses: arehaili@taibahu.edu.sa (Alrehaili AH), M.A.Walkley@leeds.ac.uk (Walkley MA), P.K.Jimack@leeds.ac.uk (Jimack PK), Matthew.Hubbard@nottingham.ac.uk (Hubbard ME) 
contains a number of examples to illustrate how theory can drive experiments and vice versa.

Generally, tumour growth is divided into three major stages:

- Avascular growth, the tumour in this stage does not get any blood supply and has small size with a clustered and structured shape. The nutrient that feeds the tumour arrives by the mechanism of diffusion.

- Angiogenesis, which is a biological process by which new blood vessels are formed, is the next stage. Moreover, the tumour still has the structured shape associated with avascular growth.

- Vascular growth is the final stage, where the tumour has access to plentiful resources (blood supply), and gets a nutrient supply from surrounding tissue. This stage can be harmful: rapid growth occurs in the mass of the tumour with unstructured shape, cells are able to exit through the walls of tumour vessel, which may then transmit through the blood stream and seed secondary tumours in other areas [3].

Most mathematical models of tumour growth focus on one particular aspect, such as avascular tumour growth (e.g. [4], [5] [6]), which is easier to validate against experiment, or vascular tumour growth (e.g. [7, 1, 3]). This work is focused upon the final stage of tumour growth, vascular tumours, which are generally the most dangerous. Continuum or discrete models can be developed which each have their own advantages. Discrete models allow detailed study of individual cells but are limited in the physical scale they can describe. Continuum models allow appropriate length scales but must approximate the cell interactions in an averaged form. At large scales the intercellular cohesion can be considered to act as a form of viscosity, so many continuum models (e.g. $[1,3,4,5])$, represent cell movement as very slow viscous flow.

In this paper, we focus on the numerical solution of a particular continuum multiphase flow model for tumour growth. The mathematical model that we study in this paper is presented by Hubbard and Byrne [1], and extends the work of Breward, Byrne and Lewis [4,3]. It is a multiphase model of vascular tumour growth in two dimensions and includes four phases : healthy cells, tumour cells, blood vessels and extracellular material, with the assumption that each phase behaves like a viscous fluid. Most of the studies in this area have used between two and four phases in 2D and 3D, often treating all of 
the phases under consideration as fluids (e.g [1]), or mixtures between fluids and solids (e.g [8]).

The most common mathematical formulations of multiphase flow require a system of partial differential equations (PDEs), which are generally both time dependent and nonlinear. There are several formulations of governing conservation equations for multiphase flow, depending on the application field (see for example in porous media [9] or in tumour growth $[1,4]$ ). In multiphase flow models, equations for conservation of mass and momentum are developed for each phase (without mixing phases). These include the terms of interaction, modelling the exchange of mass and momentum between the phases. Each of these phases is considered to have a separate volume fraction and velocity, and the sum of theses volume fractions is unity $[10,1]$.

The computational approach which is used in this work, and in [1], is a combination of the finite element method (FEM) and the finite volume method (FVM). In this two dimensional model, the hyperbolic PDEs of mass balance are discretised through an explicit, conservative, upwind, FVM scheme; whereas the momentum balance equations lead to generalised Stokes equations which are solved using a FEM scheme. Additionally, the discretization of the nutrient reaction-diffusion equation is also based upon a FEM combined with a Newton iteration to solve the resulting nonlinear algebraic equations. In [1], the discrete systems are solved using a sparse direct solver (MUMPS), which encountered restrictions even for fairly small computations. The memory and computational cost requirement for solving very large linear systems in 2D problems or for solving 3D problems may therefore cause a challenge to efficient direct solution methods. Hence the purpose of this work is to replace the use of MUMPS in [1] by designing optimal and efficient iterative algorithms for the problem of interest.

An excellent and comprehensive introduction to efficient preconditioners for the finite element solution of incompressible flow problems is provided by [11]. Mixed finite element discretizations of such problems generally lead to the need to solve a saddle point system following linearization, and the most effective preconditioners tend to respect this structure through a block matrix approach [11]. Some of the earliest examples are based upon simple block diagonal and general block preconditioners for Stokes systems ([12] and [13] respectively), with subsequent generalizations to linearized NavierStokes, as in $[14,15]$ for example. Each of these techniques is based upon the observation of [16] that, for non-singular saddle point systems, a block preconditioner based upon an exact Schur complement can lead to precondi- 
tioned systems with precisely two or three distinct eigenvalues. Consequently, the best practical preconditioners are those that can approximate the action of the Schur complement with sufficient accuracy at minimal computational cost: a philosophy that is built upon in this work.

The rest of the paper is organised as follows. In Section 2 the specific mathematical model that has been chosen for this work is described in detail. Then, the numerical schemes for this model are introduced in Section 3. Our primary contribution is to develop a novel preconditioner for the discrete form of the momentum balance equations. This is described in detail in Section 4, with results in Section 5 demonstrating close to optimal performance. To ensure the optimality of the whole solver we also introduce an optimal preconditioner for the Newton system arising from the nutrient diffusion equation. This is based upon algebraic multigrid (AMG). The third contribution of this research is to demonstrate that our preconditioning methods may be applied to a generalized model of tumour growth, with more phases, than in [1]. This is discussed in Section 6.

\section{Mathematical model}

In this section we describe the continuum mathematical model presented in [1], which is based on multiphase model for studying vascular tumour growth in two dimensions. This model includes four phases: normal/healthy and tumour cells, blood vessels and extracellular material, with the assumption that each phase behaves as a viscous fluid. There are three governing systems of equations in this model, which are: mass balance equations for the volume fraction of each phase $\left(\theta_{i}\right.$ for $\left.i=1, \ldots, 4\right)$, momentum balance equations for the flow of each phase (velocities $u_{i}$ and $v_{i}$ and pressure $p_{i}$ for $i=1, \ldots, 4)$, and a reaction-diffusion equation for the nutrient concentration (c). The nutrient, which is supplied by the blood vessels, is consumed by healthy and tumour cells, and therefore is not assumed to occupy volume in its own right. The spatial domain is denoted by $\Omega$, and the whole boundary of the domain is denoted by $\Gamma$.

The dimensional form of the mathematical model is introduced in [1]. We present here a nondimensionalised form where the independent and dependent variables have been scaled as:

$$
\vec{x}=L_{0} \vec{x}^{\prime}, \quad t=\frac{t^{\prime}}{k_{1,1}}, \quad \vec{u}_{i}=L_{0} k_{1,1} \vec{u}_{i}^{\prime}, \quad p_{i}=\Lambda p_{i}^{\prime}, \quad c=c_{v} c^{\prime},
$$


in which $L_{0}$ is a length scale, which is the initial radius of tumour seeded in the healthy tissue, $\Lambda$ is constant of the cell-cell interaction tension, $c_{v}$ is the blood vessels nutrient concentration and $k_{1,1}$ is the parameter of the birth rate for the normal cells, which used to scale the time t.

\subsection{Mass Balance Equations}

All phases considered are assumed to have the same density, so the mass balance for the healthy cells $\left(\theta_{1}\right)$, tumour cells $\left(\theta_{2}\right)$ and blood vessel $\left(\theta_{3}\right)$ volume fractions are given as follows:

$$
\begin{aligned}
\frac{\partial \theta_{1}}{\partial t^{\prime}}+\vec{\nabla}^{\prime} \cdot\left(\theta_{1} \vec{u}_{1}^{\prime}\right) & =\underbrace{\theta_{1} \theta_{4}\left(\frac{c^{\prime}}{c_{p}^{*}+c^{\prime}}\right)}_{\text {cell birth }}-\underbrace{k_{2,1}^{*} \theta_{1}\left(\frac{c_{c_{1}}^{*}+c^{\prime}}{c_{c_{2}}^{*}+c^{\prime}}\right)}_{\text {cell death }} \\
\frac{\partial \theta_{2}}{\partial t^{\prime}}+\vec{\nabla}^{\prime} \cdot\left(\theta_{2} \vec{u}_{2}^{\prime}\right) & =\underbrace{k_{1,2}^{*} \theta_{2} \theta_{4}\left(\frac{c^{\prime}}{c_{p}^{*}+c^{\prime}}\right)}_{\text {cell birth }}-\underbrace{k_{2,2}^{*} \theta_{2}\left(\frac{c_{c_{1}}^{*}+c^{\prime}}{c_{c_{2}}^{*}+c^{\prime}}\right)}_{\text {cell death }} \\
\frac{\partial \theta_{3}}{\partial t^{\prime}}+\vec{\nabla}^{\prime} \cdot\left(\theta_{3} \vec{u}_{3}^{\prime}\right) & =\underbrace{k_{3}^{*} \theta_{3} \mathcal{H}\left(\theta_{1} p_{1}^{\prime}+\theta_{2} p_{2}^{\prime}-p_{c r i t}^{*}, \epsilon_{3}^{*}\right)}_{\text {angiogenesis }}
\end{aligned}
$$

To determine the volume fraction for the extracellular phase $\left(\theta_{4}\right)$, a simplistic representation of the additional material required to create volume in the other phases during mitosis and angiogenesis and the material than remains when cells die, we use the no-voids condition:

$$
\sum_{i=1}^{4} \theta_{i}=1 .
$$

The parameters are defined as

$$
\begin{aligned}
& k_{1,2}^{*}=\frac{k_{1,2}}{k_{1,1}}, \quad k_{2,1}^{*}=\frac{k_{2,1}}{k_{1,1}}, \quad k_{3}^{*}=\frac{k_{3}}{k_{1,1}}, \quad k_{4}^{*}=\frac{k_{4}}{c_{v} k_{1,1}}, \\
& c_{p}^{*}=\frac{c_{p}}{c_{v}}, \quad c_{a}^{*}=\frac{c_{a}}{c_{v}}, \quad c_{c_{1}}^{*}=\frac{c_{c_{1}}}{c_{v}}, \quad c_{c_{2}}^{*}=\frac{c_{c_{2}}}{c_{v}}, \\
& p_{c r i t}^{*}=\frac{p_{c r i t}}{\Lambda}, \quad \epsilon_{3}^{*}=\frac{\epsilon_{3}}{\Lambda},
\end{aligned}
$$


and the smooth switch function:

$$
\mathcal{H}(p, \epsilon)=0.5\left(1+\tanh \frac{p}{\epsilon}\right), \quad \epsilon \ll 1 .
$$

In (2) $c^{\prime}$ is the nutrient concentration, $p_{i}^{\prime}$ are the pressures in each phase, $c_{p}, c_{c_{1}}, c_{c_{2}}$ denote the nutrient concentration parameters, $c_{v}$ is the nutrient concentration within the blood vessels, $k_{1,1}, k_{1,2}, k_{2,1}, k_{2,2}, k_{3}$ and $k_{4}$ are predefined rate constants, $\varepsilon$ is the volume fraction of the extracellular material at half the maximal angiogenesis rate, while $c_{a}$ is the nutrient concentration at the maximal angiogenesis rate, and $p_{c r i t}^{*}$ is the critical pressure for vessel occlusion.

Equations (2) are evolution equations for updating the volume fractions of the cells $\left(\theta_{1}, \theta_{2}\right)$ and blood vessels $\left(\theta_{3}\right)$. In the first and second equations from the system (2), $\theta_{1}$ and $\theta_{2}$ are increased during cell birth (proliferation) and decreased because of cell death, while $\theta_{4}$, the volume fraction of extracellular material, provides the required material for cell growth and birth. The rates of birth are considered to increase when the nutrient concentration increases from $0 \longrightarrow \infty$, whereas, the rate of death is assumed to decrease. To satisfy this, the parameters are chosen such $k_{1,2} \geq k_{1,1}, k_{2,1} \geq k_{2,2}$ and also $c_{c_{1}}>c_{c_{2}}$.

In the third equation from the system $(2), \theta_{3}$ is assumed to increase during angiogenesis and decrease because of vessel occlusion.

The right-hand sides in (2) are called the source and sink terms (the total of the source and sink terms must be zero in order to guarantee conservation of mass) which ensure the values of $\theta_{i}$ for $i=1,2,3,4$, belong on [0,1]. The terms relating to the birth of cells and to angiogenesis have a factor of $\theta_{i}$ and consequently ensure that $\theta_{i} \geq 0$. Also the source terms are constructed so that, because of (3), as $\theta_{i} \rightarrow 1$ other phase volume fractions that are constrained by $\theta_{i} \geq 0$, must tend to zero. The initial conditions of the phase

$\theta_{i}$ are given by $0 \leq \theta_{i}(\vec{x}, 0) \leq 1$ (with $\sum_{i=1}^{4} \theta_{i}(\vec{x}, 0)=1$ ), thus ensuring $\theta_{4}(\vec{x}, t) \geq 0$.

\subsection{Momentum Balance Equations}

The inertial terms in the incompressible Navier-Stokes equation are neglected due to the assumption that the Reynolds number is small [1]. The following equations describe momentum balance for the dimensionless phase 
velocities $\vec{u}_{i}^{\prime}$ and pressures $p_{i}^{\prime}(i=1, \ldots, 4)$ :

$\sum_{j \neq i} d_{i j}^{*} \theta_{i} \theta_{j}\left(\vec{u}_{j}^{\prime}-\vec{u}_{i}^{\prime}\right)-\theta_{i} \vec{\nabla}^{\prime} \cdot\left(\Lambda^{*} p_{i}^{\prime} \mathbf{I}\right)+\vec{\nabla}^{\prime} \cdot\left[\theta_{i}\left[\mu_{i}^{*}\left(\vec{\nabla}^{\prime} \vec{u}_{i}^{\prime}+\left(\vec{\nabla}^{\prime} \vec{u}_{i}^{\prime}\right)^{T}\right)+\lambda_{i}^{*}\left(\vec{\nabla}^{\prime} \cdot \vec{u}_{i}^{\prime}\right) \mathbf{I}\right]\right]=0$,

in which the first term models the interphase drag and the remaining terms represent the viscous stresses for this phase.

The incompressibility condition implies that

$$
\sum_{i=1}^{4} \vec{\nabla}^{\prime} \cdot\left(\theta_{i} \vec{u}_{i}^{\prime}\right)=0
$$

where

$$
p_{1}^{\prime}=p_{2}^{\prime}=p_{4}^{\prime}+\Sigma^{\prime}(\theta), \quad p_{3}^{\prime}=\frac{p_{3}^{*}}{\Lambda} .
$$

The term $\Sigma^{\prime}(\theta)$ describes the pressure in the cell resulting from cell-cell interaction:

$$
\Sigma^{\prime}(\theta)= \begin{cases}\frac{\left(\theta-\theta^{*}\right)}{(1-\theta)^{2}} & \text { if } \theta \geq \theta^{*} \\ 0 & \text { if } \theta<\theta^{*},\end{cases}
$$

where $\theta^{*}$ is a given value, known as the cells natural density and $\theta=\theta_{1}+\theta_{2}$, $\theta^{*}$. Furthermore, in (4),

$$
\begin{gathered}
d_{i j}^{*}=\frac{d_{i j}}{d_{12}}, \Lambda^{*}=\frac{\Lambda}{d_{12} k_{1,1} L_{0}^{2}}, \\
\mu_{i}^{*}=\frac{\mu_{i}}{d_{12} L_{0}^{2}}, \quad \lambda_{i}^{*}=\frac{\lambda_{i}}{d_{12} L_{0}^{2}},
\end{gathered}
$$

in which $d_{i j}^{*}$ is the drag coefficient, $d_{i j}=d_{j i}$ for $i, j=1,2,3,4$, and $i \neq j$, $\mu_{i}$ denotes the dynamic shear and $\lambda_{i}$ denotes the bulk viscosities, which are related through $\lambda_{i}=-\frac{2}{3} \mu_{i}$.

The momentum balance equations (4), do not depend on time and are linear in ${\overrightarrow{u_{i}}}^{\prime}$ and $p_{4}^{\prime}$. Furthermore, $p_{3}^{\prime}$ is assumed to be constant $\left(p_{3}^{\prime}=\frac{p_{3}^{*}}{\Lambda}\right)$, where $p_{3}^{*}$ is the externally-imposed pressure which is assumed constant. Hence, the equations (4) update the velocities $\overrightarrow{u_{i}}$ (four velocities in x-direction and four velocities in $\mathrm{y}$-direction) and only the pressure $p_{4}^{\prime}$.

\subsection{Reaction Diffusion Equation}

The governing equation for the nutrient/oxygen concentration $c^{\prime}$, can be written as 


$$
\begin{aligned}
D_{c}^{*} \nabla^{\prime 2} c^{\prime} & =\underbrace{\theta_{3}\left(1-c^{\prime}\right)}_{\text {replenishment }} \underbrace{-k_{6,1}^{*} \theta_{1} c^{\prime}-k_{6,2}^{*} \theta_{2} c^{\prime}}_{\text {baseline consumption }} \\
& -\underbrace{k_{7,1}^{*} \theta_{1} \theta_{4}\left(\frac{c^{\prime}}{c_{p}^{*}+c^{\prime}}\right)-k_{7,2}^{*} \theta_{2} \theta_{4}\left(\frac{c^{\prime}}{c_{p}^{*}+c^{\prime}}\right)}_{\text {consumption due to cell birth }},
\end{aligned}
$$

in which

$$
\begin{array}{ll}
D_{c}^{*}=\frac{D_{c}}{k_{5} L_{0}^{2}}, & k_{6,1}^{*}=\frac{k_{6,1}}{k_{5}}, \quad k_{6,2}^{*}=\frac{k_{6,2}}{k_{5}}, \\
k_{7,1}^{*}=\frac{k_{7,1}}{c_{v} k_{5}}, & k_{7,2}^{*}=\frac{k_{7,2}}{c_{v} k_{5}} .
\end{array}
$$

Here $D_{c}^{*}$ is the diffusion coefficient, $\left(k_{5}, k_{6,1}, k_{6,2}, k_{7,1}, k_{7,2}\right)$ are pre-defined rate

constants, with assumed $\frac{k_{7,1}}{k_{7,2}}=\frac{k_{1,1}}{k_{1,2}}$. The equation (6) does not contain a time derivative, and is nonlinear in the single variable $c^{\prime}$.

\section{Numerical methods}

In [1] the computational approach is a combination of FEM and FVM on unstructured triangular meshes. In summary, at each time step:

- A cell-centred finite volume scheme is used to approximate the mass balance equations (2) to update the cell-average $\theta_{i}$ for three phases while the no-void condition (3) is used to update the fourth phase.

- A stable Galerkin finite element scheme, with Taylor-Hood elements, is used to approximate the incompressible momentum balance equations (4) to solve for new $\vec{u}_{i}$ and $p_{i}$.

- A standard Galerkin finite element scheme with linear elements approximates the reaction-diffusion equation (6) to update the nutrient c. Note that this is solved on a finer mesh than for the previous two steps, see [1] for details.

The detail of these numerical schemes are presented in the following subsections. 


\subsection{Mass Balance Equations}

Equation (2) is a set of hyperbolic mass balance equations which are time dependent PDEs. These equations are approximated using an explicit Euler solver in time with a standard cell centred finite volume scheme in space. The integral form for the mass balance equation is given by

$$
\int_{\Delta} \frac{\partial \theta_{i}}{\partial t} d \vec{x}+\oint_{\partial \Delta}\left(\theta_{i} \vec{u}_{i}\right) \cdot \vec{n} d s=\int_{\Delta} q_{i} d \vec{x}, \quad i=1, \ldots, 3
$$

where $\Delta$ is the control volume, $q_{i}$ represents the source/sink terms, $\partial \Delta$ is the boundary of the control volume, and $\vec{n}$ is the outward-pointing unit normal to the boundary. In [1] a cell-centred MUSCL approach is used, which is a conservative, upwind, finite volume scheme. This is used to update $\theta_{1}, \theta_{2}$, and $\theta_{3}$ in time, while the no-voids condition (3) is used to update $\theta_{4}$. The fluxes $\theta_{i} \vec{u}_{i}$ are approximated by using a standard upwind scheme [17]. So, the discrete equation for each triangle can be written as

$$
\bar{\theta}_{i}^{n+1}=\bar{\theta}_{i}^{n}-\frac{\Delta t}{|\Delta|} \sum_{k=1}^{3}\left(\theta_{i}^{n} \overrightarrow{u_{i}^{n}}\right)_{k}^{*} \cdot \overrightarrow{n_{k}}+\Delta t\left(q_{i}^{n}\right)^{*}, i=1, \ldots, 4 .
$$

in which $n$ denotes the time level, $\Delta t$ is the time step size, $|\Delta|$ is the control volume area, and $\overrightarrow{n_{k}}$ is the outward-pointing unit normal to the edge of the cell opposite the vertex $\mathrm{k}$.

To define the boundary condition, the volume fraction $\theta_{i}$ is described for

each phase on the inflow section $\Gamma_{i}^{\text {inflow }}$ for $t \geq 0$. These inflow sections belong to the domain boundary $\Gamma$ where $\overrightarrow{u_{i}} \cdot \vec{n}<0$, where $\vec{n}$ is the outwardpointing unit normal to $\Gamma$.

\subsection{Momentum Balance Equations}

The momentum balance equations (4), do not depend on time, and they are linear in $\overrightarrow{u_{i}}$ and $p_{4}$. The weak form is obtained using a Galerkin finite element scheme with Taylor-Hood elements to yield

$$
\begin{gathered}
\oint_{\partial \Omega} \omega^{q} \theta_{i} \sigma_{i} \cdot \vec{n} d s-\int_{\Omega} \vec{\nabla} \omega^{q} \cdot \theta_{i} \sigma_{i} d \vec{x}+\int_{\Omega} \omega^{q} \vec{F}_{i} d \vec{x}=0, \quad i=1, \ldots, 4, \\
\int_{\Omega} \omega^{l} \sum_{i=1}^{4} \vec{\nabla} \cdot\left(\theta_{i} \vec{u}_{i}\right) d \vec{x}=0,
\end{gathered}
$$


where $\omega^{l}$ and $\omega^{q}$ are the standard linear and quadratic Lagrange test functions respectively, $\sigma_{i}$ are the stresses in each individual phase

$$
\sigma_{i}=-p_{i} \mathbf{I}+\mu_{i}\left(\vec{\nabla} \vec{u}_{i}+\left(\vec{\nabla} \vec{u}_{i}\right)^{T}\right)+\lambda_{i}\left(\vec{\nabla} \cdot \vec{u}_{i}\right) \mathbf{I}
$$

and $\vec{F}_{i}$ are the momentum sources

$$
\vec{F}_{i}=p_{i} \mathbf{I} \vec{\nabla} \theta_{i}+\sum_{j=1, j \neq i}^{4} d_{i j} \theta_{i} \theta_{j}\left(\vec{u}_{j}-\vec{u}_{i}\right), \quad i=1, \ldots, 4
$$

The velocities $\overrightarrow{u_{i}}$ and pressures $\mathrm{p}$ are approximated by using piecewise polynomials, which are written using the trial function as

$$
\overrightarrow{u_{i}} \approx \sum_{k=1}^{N_{q}}\left(\overrightarrow{u_{i}}\right)_{k} \omega_{k}^{q}, \quad \quad p \approx \sum_{k=1}^{N_{l}} p_{k} \omega_{k}^{l},
$$

in which $N_{q}$ and $N_{l}$ are, respectively, the numbers of degrees of freedom related to the quadratic and linear Lagrange elements.

There are two possible boundary conditions used for the momentum balance equations, which are Dirichlet condition on $\overrightarrow{u_{i}}$ or $\sigma_{i} \cdot \vec{n}$ specified. Here, the boundary conditions imposed are zero normal stress $\sigma_{i} \cdot \vec{n}=0$ for the healthy cell, tumour cell and blood vessel phases, and zero velocity for the extracellular phase $\vec{u}_{4}=0$.

Applying this method to discretize (10) and (11) using trial functions (12) leads to a matrix system that may be expressed in the block-matrix form:

$$
\left(\begin{array}{ccccccccc}
k_{x x 11} & k_{x y 11} & k_{x x 12} & 0 & k_{x x 14} & 0 & k_{x x 13} & 0 & C_{x 1} \\
k_{y x 11} & k_{y y 11} & 0 & k_{y y 12} & 0 & k_{y y 14} & 0 & k_{y y 13} & C_{y 1} \\
k_{x x 22} & 0 & k_{x x 22} & k_{x y 22} & k_{x x 24} & 0 & k_{x x 23} & 0 & C_{x 2} \\
0 & k_{y y 21} & k_{y x 22} & k_{y y 22} & 0 & k_{y y 24} & 0 & k_{y y 23} & C_{y 2} \\
k_{x x 41} & 0 & k_{x x 42} & 0 & k_{x x 44} & k_{x y 44} & k_{x x 43} & 0 & C_{x 4} \\
0 & k_{y y 41} & 0 & k_{y y 42} & k_{y x 44} & k_{y y 44} & 0 & k_{y y 43} & C_{y 4} \\
k_{x x 31} & 0 & k_{x x 32} & 0 & k_{x x 34} & 0 & k_{x x 33} & k_{x y 33} & 0 \\
0 & k_{y y 3} & 0 & k_{y y 32} & 0 & k_{y y 3} & k_{y x 33} & k_{y y 33} & 0 \\
B_{x 1}^{T} & B_{y 1}^{T} & B_{x 2}^{T} & B_{y 2}^{T} & B_{x 4}^{T} & B_{y 4}^{T} & B_{x 3}^{T} & B_{y 3}^{T} & 0
\end{array}\right)\left(\begin{array}{c}
u_{x 1} \\
u_{y 1} \\
u_{x 2} \\
u_{y 2} \\
u_{x 4} \\
u_{y 4} \\
u_{x 3} \\
u_{y 3} \\
p_{4}
\end{array}\right)=\left(\begin{array}{c}
f x_{1} \\
f y_{1} \\
f x_{2} \\
f y_{2} \\
f x_{4} \\
f y_{4} \\
f x_{3} \\
f y_{3} \\
0
\end{array}\right)
$$

The coefficient matrix A has $9 \times 9$ block structure with 8 vectors of velocity variables and 1 vector of pressure variables. The first 8 block rows are obtained from equations (10). Further details of the discretisation are provided in the reference [18]. Each two rows express the $\mathrm{x}$ and $\mathrm{y}$ directions of momentum of each phase, starting from the healthy phase, tumour phase, extracellular phase and blood vessels phase. The final block row is obtained 
from equation (11), the continuity equation. The number of unknowns in this linear system is $N=8 N_{q}+N_{l}$.

In [1] the resulting large sparse system of linear equations is solved using the direct solution package MUMPS [19]. The system is nonsymmetric and indefinite and has similarities with the standard system arising from a mixed FEM discretization of Stokes' equation for standard, single phase, incompressible flow.

\subsection{Reaction Diffusion Equation}

Equation (6) is the quasi/steady state, reaction-diffusion equation. This equation is a steady state nonlinear PDE, and is approximated using a standard Galerkin finite element scheme with linear elements to get:

$$
\int_{\partial \Omega} \omega^{l_{f}} D_{c} \vec{\nabla} c \cdot \vec{n} d s-\int_{\Omega} \vec{\nabla} \omega^{l_{f}} \cdot D_{c} \vec{\nabla} c d \vec{x}-\int_{\Omega} \omega^{l_{f}} q_{c} d \vec{x}=0,
$$

in which $D_{c}$ denotes the diffusion coefficient, which is assumed to be constant, $\omega^{l_{f}}$ is linear test function defined on the finer mesh, which is used to update the volume fractions of the phases, and $q_{c}$ is a source term, which is a nonlinear function of the the nutrient concentration $\mathrm{c}$.

The nutrient concentration $\mathrm{c}$ is written using the trial functions as:

$$
c \approx \sum_{k=1}^{N_{l_{f}}} c_{k} \omega_{k}^{l_{f}}
$$

There are two possible boundary conditions used for the reaction diffusion equation, which are Dirichlet conditions $c$ or Neumann conditions $\vec{\nabla} c . \vec{n}$. In this work $\vec{\nabla} c \cdot \vec{n}=0$ is specified on the boundary.

Here, FEM leads to a nonlinear system of equations with a sparse Jacobian which is solved using a Newton method to linearize it. The MUMPS sparse direct algorithm is again used to solve the linear system of equations at each nonlinear iteration in [1].

\subsection{Meshes for discretization}

In this work two different triangular meshes are used for each solve: (i) the original mesh, on which the momentum equations are solved; and (ii) a uniform refinement of this original mesh, on which the mass equations and the reaction-diffusion equation are solved. Because we use the Taylor-Hood FEM 
scheme to approximate the momentum equations, each edge of this original mesh has velocity unknowns at its midpoint due to the quadratic Lagrange polynomial basis functions used for the velocity unknowns. Consequently, the vertices of the uniformly refined mesh correspond to the locations of the velocity degree of freedom: hence the mass and concentration unknowns on the refined mesh correspond to the velocity unknowns [1].

\subsection{Solution of linear and nonlinear systems}

The efficiency of the numerical scheme used in [1] is limited by the suboptimal algorithms used to solve the linear and nonlinear algebraic systems

resulting from the FEM approximations. The use of a sparse direct solver is sub-optimal and leads to a very significant time and memory requirements to solve the momentum balance equations at each time-step. Increasing the mesh resolution or the number of phases present in the $2 \mathrm{D}$ model adds significant extra memory and CPU overhead with this sparse direct solver.

In the remainder of this paper we introduce improved solution algorithms to reduce the $\mathrm{CPU}$ time and memory requirements. An AMG preconditioned GMRES method is used to solve the discrete algebraic linear system that arises from approximation the reaction-diffusion equation (6) at each Newton iteration. Furthermore, a new block preconditioned GMRES method is used to solve the discrete system arising from the momentum balance equations. This is described in full in the following section.

Simpler preconditioners, based on incomplete factorisation, have been tested and a wider comparison is reported elsewhere [18].

\section{A pseudo-optimal preconditioner for the momentum equation}

In general, the block-matrix system (13) can be written as:

$$
\underbrace{\left(\begin{array}{cc}
K & C \\
B^{T} & 0
\end{array}\right)}_{A}\left(\begin{array}{l}
U \\
P
\end{array}\right)=\left(\begin{array}{l}
f \\
0
\end{array}\right),
$$

in which $\mathrm{K}$ is $8 \times 8$ block matrix that includes all the $\mathrm{k}$ blocks, $\mathrm{C}$ is the $8 \times 1$ block matrix, which includes all the $\mathrm{C}$ blocks, and $B^{T}$ is the $1 \times 8$ block matrix that includes all the $B^{T}$ blocks. Also, $\mathrm{U}$ denotes all 8 velocity-vectors and $\mathrm{P}$ is $p_{4}$.

It is clear that this problem is Stokes-like (though it is far more complex than a standard Stokes problem). In this model, matrix A is large and sparse 
and the system should be solved iteratively. The discrete system is nonsymmetric, hence to solve this problem iteratively we choose a preconditioned GMRES method. Efficient block preconditions for this class of problem are known, provided subsystems involving the matrix $\mathrm{K}$ and the Schur complement $S=B^{T} K^{-1} C$ can be solved efficiently [20].

\subsection{The block preconditioning}

This subsection presents how we have developed a new efficient block preconditioner for the system (13). The new preconditioner is created using only upper triangular blocks with the bottom right block taking the form of the mass matrix for the finite elements used to approximate the pressure, mp such a preconditioner can be written as follows:

$$
P=\left(\begin{array}{ccccccccc}
k_{x x 11} & k_{x y 11} & k_{x x 12} & 0 & k_{x x 14} & 0 & k_{x x 13} & 0 & C_{x 1} \\
0 & k_{y y 11} & 0 & k_{y y 12} & 0 & k_{y y 14} & 0 & k_{y y 13} & C_{y 1} \\
0 & 0 & k_{x x 22} & k_{x y 22} & k_{x x 24} & 0 & k_{x x 23} & 0 & C_{x 2} \\
0 & 0 & 0 & k_{y y 2} & 0 & k_{y y 24} & 0 & k_{y y 23} & C_{y 2} \\
0 & 0 & 0 & 0 & k_{x x 44} & k_{x y 44} & k_{x x 43} & 0 & C_{x 4} \\
0 & 0 & 0 & 0 & 0 & k_{y y 44} & 0 & k_{y y 43} & C_{y 4} \\
0 & 0 & 0 & 0 & 0 & 0 & k_{x x 33} & k_{x y 33} & 0 \\
0 & 0 & 0 & 0 & 0 & 0 & 0 & k_{y y 33} & 0 \\
0 & 0 & 0 & 0 & 0 & 0 & 0 & 0 & m p
\end{array}\right) .
$$

In order to be able to invert the preconditioner as efficiently as possible two further steps are taken. Firstly, the diagonal blocks are solved only approximately, using just one V-cycle of the algebraic multigrid method, and secondly the diagonal of $\mathrm{mp}$ is used in the final diagonal block in place of mp. We solve the preconditioning problem through a block-based backsubstitution. In practice, one AMG V-cycle is used with Gauss Seidel smoothing and 2 pre-and post-smoothing iterations to solve the diagonal blocks.

Before presenting the numerical results that are obtained from using the proposed preconditioner, the eigenvalues of the preconditioner are considered. One of the motivations for computing the eigenvalues for this problem is to understand the quality of the preconditioner for the GMRES solution [21]. If the eigenvalues of the matrix A have a large spread, then the GMRES method typically has slow convergence [21]. Moreover, a good preconditioner should be a good approximation to the original matrix A and the eigenvalues of the preconditioned system should be clustered in small groups and be bounded away from zero and infinity.

For these results we have exactly inverted the diagonal blocks of the preconditioner $\mathrm{P}$, using the direct solver, to fined the eigenvalues of the preconditioned system $A P^{-1}$. The eigenvalues of the resulting preconditioned systems are bounded in a small range compared to the eigenvalues of the 
matrix A, which are spread widely over the region (see Table 1). Furthermore, the spread of the eigenvalues of the original matrix increases with the problem size, while the range of the eigenvalues of the preconditioned systems is almost fixed for all problems sizes. In addition, a plot of the eigenvalues in the complex plane shows that using the preconditioning technique makes the majority of the eigenvalues cluster in a small range around 1, with just a small number of isolated eigenvalues near to the origin.

Table 1: Minimum and maximum eigenvalues of the coefficient matrix A and preconditioned matrix $A P^{-1}$, computed using a sequence of uniform grids (see Figure 1).

\begin{tabular}{ccccc}
\hline Grid & MIN $\lambda(A)$ & MAX $\lambda(A)$ & MIN $\lambda\left(A P^{-1}\right)$ & MAX $\lambda\left(A P^{-1}\right)$ \\
\hline $9^{2}$ & 0.0013 & 85.6117 & 0.0044 & $1.0182+0.0037 \mathrm{i}$ \\
$17^{2}$ & $6.9685 \mathrm{e}-07$ & 101.1776 & 0.0045 & $1.0146+0.0002 \mathrm{i}$ \\
$33^{2}$ & $4.7706 \mathrm{e}-08$ & 113.4432 & 0.0046 & $1.0154+0.0001 \mathrm{i}$ \\
\hline
\end{tabular}

\subsection{Optimal performance}

Before introducing the computational and memory costs for the whole tumour model with our proposed approach based on preconditioned GMRES, we present here the effect of our new block preconditioning on the time required for solving the momentum equation system.

The performance for solving the discrete linear system arising from discretization of the momentum equations are presented in the next table. Table 2 presents the running time needed to solve the linear system using MUMPS compared with our solver. It can be observed that MUMPS has a computational complexity that is significantly worse than linear. By replacing this solver with P-preconditioned GMRES, the performance of the solution improved significantly and the running time scales approximately linearly with problem size. 
Table 2: The average running times (in seconds), over 1000 time steps, required for solving the linear algebraic system using MUMPS and our solver on fully unstructured grids: $\mathrm{N}$ is the number of unknowns, GI is GMRES iterations, computed using a sequence of unstructured grids (see Figure 1).

\begin{tabular}{c|c|cc} 
& MUMPS & \multicolumn{2}{|c}{ Our solver } \\
\hline $\mathrm{N}$ & Average time per step & Average GI & Average time per step \\
\hline 76237 & 9.01380657199 & 35.138 & 2.12991429 \\
302252 & 64.09243820 & 39.811 & 8.9229566389 \\
1203643 & - & 41.901 & 38.244552119 \\
\hline
\end{tabular}

To conclude, P-preconditioned GMRES is a superior solver, achieving almost optimal solution times on unstructured grids. Moreover, the new solution scheme reduced the memory that is required to solve the problem.

\section{Numerical results}

This section considers the computational and memory costs for the whole tumour model with our proposed approach of preconditioned GMRES with AMG preconditioning for solving the discrete reaction diffusion equation system (6) and our new preconditioning, P, with GMRES for solving the discrete momentum equations system (2). The numerical experiments presented in this work have been carried out on a standard desktop machine with 16 GB of memory.

We use two different sequences of grids to solve the problem. The first is a circular domain of radius 16 with unstructured triangular grids. The second is a square domain with a regular triangular grid with equal diameter to the circle (see Figure 1). We start with the same grid from [1] that has the number of nodes 2349 and the number of elements 4539 . We then apply uniform mesh refinements to obtain a sequence of finer grids, each with approximately four times more unknowns than the previous. 
Figure 1: Uniform and unstructured grids used for simulations.
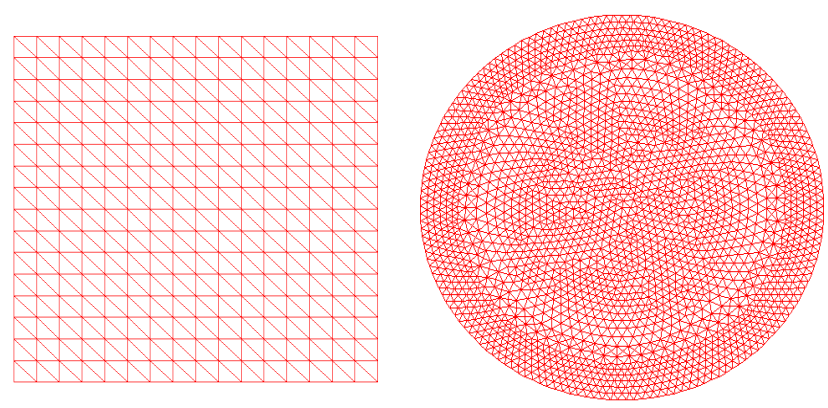

The initial conditions for the whole model for simulation of a single tumour seeded in the centre of healthy tissue can be given as follows:

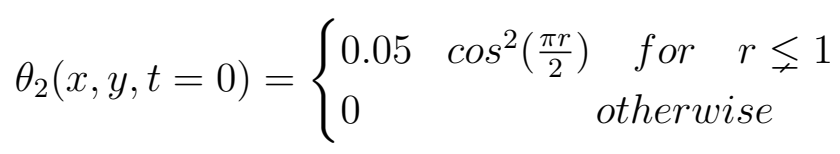

in which $r=\sqrt{x^{2}+y^{2}}$.

- $\theta_{1}(x, y, 0)=0.6-\theta_{2}(x, y, 0), \theta_{3}=0.0174978$ and $\theta_{4}=0.3825022$.

- Each phase has zero velocity.

- $p_{3}=0$ and $p_{4}=0$, then $p_{1}=p_{2}=0$ due to $\theta_{1}=\theta^{*}$ in equation (5).

- The nutrient concentration $c^{\prime}=0.2532031$ everywhere.

The boundary conditions for the whole model are:

- For the system of equations (2) we impose only inflow conditions on the section of the boundary $\Gamma_{i}^{\text {inflow }}$ on which $\vec{u}_{i}^{\prime} \cdot \vec{n}<0$

$$
\theta_{i}=\theta_{i}^{\infty}, \quad i=1, \ldots, 4
$$

- For the system of equations (4) we impose

$$
\sigma_{i}^{\prime} \cdot \vec{n}=0, \quad i=1, . ., 3 \quad \text { and } \quad \vec{u}_{4}^{\prime}=0 .
$$

throughout $\Gamma$, where $\sigma_{i}^{\prime}$ are the stresses in each individual phase. 
- For the system of equations (6) we impose

$$
\vec{\nabla}^{\prime} c^{\prime} \cdot \vec{n}=0
$$

where $\vec{n}$ is the unit outward-pointing normal to $\Gamma$. We used the values of parameters that are shown in Table 1 in [1] .

In all the experiments we used the mathematical and computational models that are written in the FORTRAN language by Hubbard and Byrne [1] modified to use the iterative solvers developed here. In addition, we make use of the software implementation of iterative methods that are available in the Harwell Subroutine Library (HSL) [22]. This includes: HSL-MI20 for AMG preconditioner [23] and HSL-MI24 for GMRES method. For testing purposes all the results in this paper are obtained using a fixed time step size $\Delta t=0.25$ with the number of steps 1000 .

For the AMG-preconditioned GMRES method, one AMG V-cycle is used with two pre- and post-smoothing iterations. For this nonlinear system the full AMG algorithm must be applied at each Newton iteration. The GaussSeidel method is used as smoother. Here, the restart parameter $(\mathrm{m})$ and the maximum number of GMRES iterations are given the same value (i.e. full GMRES is used). Also, the absolute and relative convergence tolerances are given respectively 0.0 and $1 e-3$.

For the block-preconditioned GMRES method, solving this linear system can be split into a single step that forms the AMG hierarchy of grids followed by application of the AMG solver at each time step. The restart parameter (m) of GMRES and the maximum number of GMRES iterations (maxGI) are given the same value. Also, the absolute and relative tolerances for the GMRES are set to $1 e-10$ and $1 e-3$ respectively. The blocks in the diagonal of our preconditioner $\mathrm{P}$ are solved using one $\mathrm{V}$-cycle of AMG with two preand post-smoothing stages.

For comparison the original solver was run for the same problems and CPU time and memory use were collected. Results are shown in Table 3 for the circular domain. As expected, the MUMPS solver can not be used to solve large problems due to memory requirement. Furthermore, our solver achieved much faster times and required much less memory (see Table 3). The time and the memory requirement for our preconditioner behave almost optimally as $\mathcal{O}(N)$. 
Table 3: The CPU time and percentage of memory cost for solving the whole model on fully unstructured grids using the MUMPS and our solvers: $\mathrm{N}$ is the number of unknowns in the discrete momentum system.

\begin{tabular}{c|cc|cc} 
& \multicolumn{2}{|c|}{ MUMPS } & \multicolumn{2}{c}{ Our solver } \\
\hline $\mathrm{N}$ & MEM (\%) & Time & MEM $(\%)$ & Time \\
\hline 76237 & 3.3 & $194 \mathrm{~m} 58 \mathrm{~s}$ & 2.4 & $37 \mathrm{~m} 22 \mathrm{~s}$ \\
302252 & 18.4 & $1084 \mathrm{~m} 4 \mathrm{~s}$ & 8 & $156 \mathrm{~m} 47 \mathrm{~s}$ \\
1203643 & - & - & 27.5 & $672 \mathrm{~m} 28 \mathrm{~s}$ \\
\hline
\end{tabular}

Similar performance is found when another type of the domain is used. The results of the application of the model on a square domain using regular triangular grids, such as in Figure 1, are shown in Table 4 where the optimally of the results is very clearly observed.

Table 4: The CPU time and percentage of memory cost for solving the whole model on regular grids using preconditioned GMRES: the linear system solved using $\mathrm{p}$ and the nonlinear system solved using AMG preconditioning.

\begin{tabular}{cccc}
\hline Grid & $\mathrm{N}$ & MEM $(\%)$ & Time \\
\hline $33^{2}$ & 34889 & 1 & $15 \mathrm{~m} 16 \mathrm{~s}$ \\
$65^{2}$ & 137353 & 3.4 & $58 \mathrm{~m} 22 \mathrm{~s}$ \\
$129^{2}$ & 545033 & 12.7 & $246 \mathrm{~m} 53 \mathrm{~s}$ \\
$257^{2}$ & $2,171,401$ & 48.5 & $998 \mathrm{~m} 10 \mathrm{~s}$ \\
\hline
\end{tabular}


Figure 2: From the top row to bottom row: the evolution of the volume fraction of tumour cells $\theta_{2}$, the flux of tumour cells $\theta_{2} \vec{u}_{2}^{\prime}$, the pressures of healthy and tumour cells $p_{1}=p_{2}$, and the nutrient concentration c. Increasing time from left to right: $t=100,200$ and 300. The number of unknowns in the momentum system is 76237 .

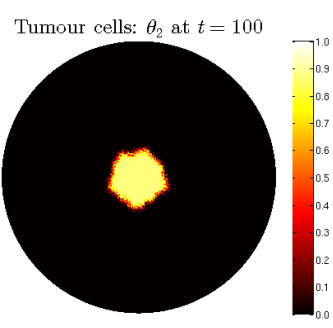

Tumour cells: $\theta_{2} \vec{u}_{2}$ at $t=100$
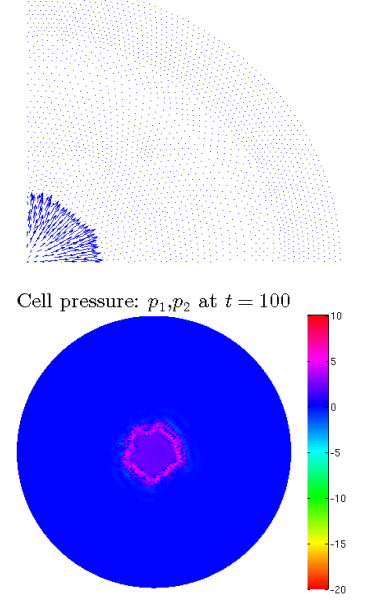

Nutrient: $c^{\prime}$ at $t=100$

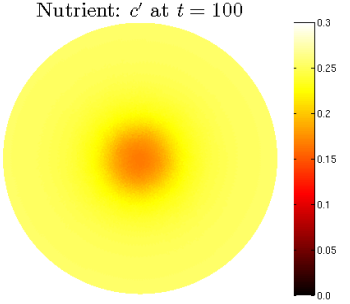

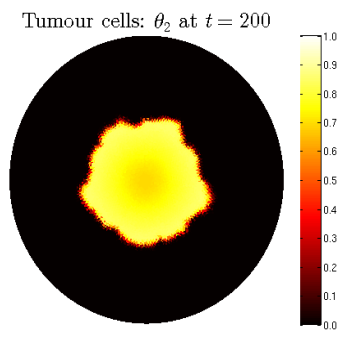

Tumour cells: $\theta_{2} \vec{u}_{2}$ at $t=200$
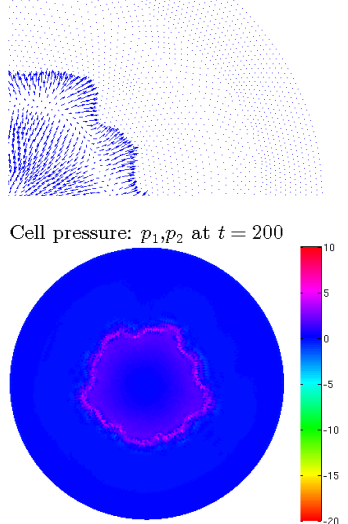

Nutrient: $c^{\prime}$ at $t=200$

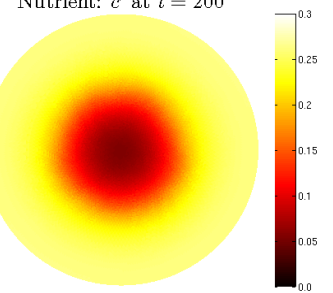

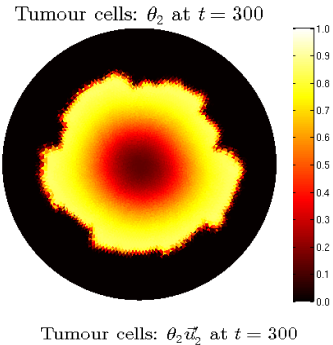

Tumour cells: $\theta_{2} \vec{u}_{2}$ at $t=300$
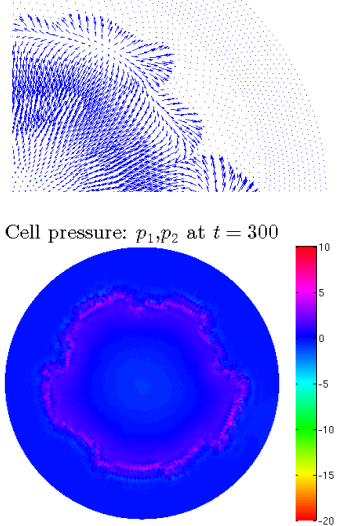

Nutrient: $c^{\prime}$ at $t=300$

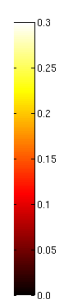

The numerical simulations in Figure 2 show how the volume fraction of tumour phase, the evolution of the phase flux, pressures and the nutrient concentration develop with the time on the circle domain. Initially, the simulation starts with a seed cluster of tumour cells in the middle of healthy tissue (not shown). This cluster of tumour extends over time to produce a high tumour cell density around the initial seed. The volume fraction of tumour cells grows rapidly and reaches a maximum value, which is in the 
range between 0.8 and 0.9 . Then the tumour cells spread out and die in the centre, forming necrotic core due to the local nutrient concentration being insufficient to maintain the tumour cells. It can be noted that the tumour has an imperfect circle shape, due to the unstructured grid causing the radial symmetry to be broken.

The proliferation and death rates of the tumour cells are assumed to be double and half the values of the proliferation and death rates of the healthy cells respectively. So, the tumour cells grow faster and die slower than the healthy cells. In this case, the tumour cells absorb the extracellular material during their growth. This leads to a fall in the extracellular material $\theta_{4}$ and therefore leads to decreasing the healthy cells' birth rate. Also, when the tumour cells grow faster than the healthy cells that leads to the tumour cells pushing the healthy cells in front and replacing the volume by the extracellular material. Furthermore, the high tumour cells density generate high pressures which leads to the occlusion of the blood vessels and therefore restricts the supply of the nutrient inside the tumour.

\section{Generalization to a greater number of phases}

In this section we demonstrate that the optimal preconditioning approach that we developed for the four-phase model with a nutrient may be naturally extended to more general multiphase models. This is achieved by considering two extensions of [1]. Firstly, the mathematical model of vascular tumour growth is extended to include drug delivery, diffusion and uptake. Secondly, the mathematical model is extended to simulate five phases. The extra phase in our mathematical model is generated by splitting the tumour phase into two different phases: one with high susceptibility to the drug (HS) and other with low susceptibility to the drug (LS). So, the five phases in this model are: healthy cells, LS tumour cells, HS tumour cells, blood vessels and extracellular material. In this paper our main objective in introducing the five-phase model is to show that it is possible to extend our momentum equation preconditioner, $\mathrm{P}$, to a system with more phases.

Briefly, the generalization of the momentum balance equation for the new model can be written as follow

$$
\sum_{j \neq i} d_{i j}^{*} \theta_{i} \theta_{j}\left(\vec{u}_{j}^{\prime}-\vec{u}_{i}^{\prime}\right)-\theta_{i} \vec{\nabla}^{\prime} .\left(\Lambda^{*} p_{i}^{\prime} \mathbf{I}\right)+\vec{\nabla}^{\prime} \cdot\left[\theta_{i}\left[\mu_{i}^{*}\left(\vec{\nabla}^{\prime} \vec{u}_{i}^{\prime}+\left(\vec{\nabla}^{\prime} \vec{u}_{i}^{\prime}\right)^{T}\right)+\lambda_{i}^{*}\left(\vec{\nabla}^{\prime} \cdot \vec{u}_{i}^{\prime}\right) \mathbf{I}\right]\right]=0 .
$$


for $i, j=1,2,3,4,5$. The incompressibility condition implies that

$$
\sum_{i=1}^{5} \vec{\nabla}^{\prime} \cdot\left(\theta_{i} \vec{u}_{i}^{\prime}\right)=0
$$

where

$$
p_{1}^{\prime}=p_{2}^{\prime}=p_{5}^{\prime}=p_{4}^{\prime}+\sum^{\prime}(\theta), \quad p_{3}^{\prime}=\frac{p_{3}^{*}}{\Lambda} .
$$

The momentum balance equations (18) is linear in $\vec{u}_{i}^{\prime}$ and $p_{4}^{\prime}$, and is not time dependent. Moreover, $p_{3}^{\prime}$ is assumed to be constant $\left(p_{3}^{\prime}=\frac{p_{3}^{*}}{\Lambda}\right)$, where $p_{3}^{*}$ is the externally-imposed pressure and it is assumed constant. Hence, the equations (18) update the velocities $\overrightarrow{u_{i}}$ (five velocities in x-direction and five velocities in y-direction) and only the pressure $p_{4}^{\prime}$.

The numerical approach for the discretization of the momentum equations is essentially the same as for four phase system considered previously, based upon Taylor-Hood elements. The boundary conditions used are zero normal stress $\sigma_{i} \cdot \vec{n}=0$ for four phases: healthy cells, LS tumour cells, HS tumour cells and blood vessels; and zero velocity for the extracellular phase $\vec{u}_{4}=0$.

Following the same approach as for the four phase system discretization yields a similar block-matrix system for the discrete momentum equations in our new model:

$$
\left(\begin{array}{ccccccccccc}
k_{x x 11} & k_{x y 11} & k_{x x 12} & 0 & k_{x x 15} & 0 & k_{x x 14} & 0 & k_{x x 13} & 0 & C_{x 1} \\
k_{y x 11} & k_{y y 11} & 0 & k_{y y 12} & 0 & k_{y y 15} & 0 & k_{y y 14} & 0 & k_{y y 13} & C_{y 1} \\
k_{x x 21} & 0 & k_{x x 22} & k_{x y 22} & k_{x x 25} & 0 & k_{x x 24} & 0 & k_{x x 23} & 0 & C_{x 2} \\
0 & k_{y y 21} & k_{y x 22} & k_{y y 22} & 0 & k_{y y 25} & 0 & k_{y y 24} & 0 & k_{y y 23} & C_{y 2} \\
k_{x x 51} & 0 & k_{x x 52} & 0 & k_{x x 55} & k_{x y 55} & k_{x x 54} & 0 & k_{x x 53} & 0 & C_{x 5} \\
0 & k_{y y 51} & 0 & k_{y y 52} & k_{y x 55} & k_{y y 55} & 0 & k_{y y 54} & 0 & k_{y y 53} & C_{y 5} \\
k_{x x 41} & 0 & k_{x x 42} & 0 & k_{x x 45} & 0 & k_{x x 44} & k_{x y 44} & k_{x x 43} & 0 & C_{x 4} \\
0 & k_{y y 41} & 0 & k_{y y 42} & 0 & k_{y y 45} & k_{y x 44} & k_{y y 44} & 0 & k_{y y 43} & C_{y 4} \\
k_{x x 31} & 0 & k_{x x 32} & 0 & k_{x x 35} & 0 & k_{x x 34} & 0 & k_{x x 33} & k_{x y 33} & 0 \\
0 & k_{y y 31} & 0 & k_{y y 32} & 0 & k_{y y 35} & 0 & k_{y y 34} & k_{y x 33} & k_{y y 33} & 0 \\
B_{x 1}^{T} & B_{y 1}^{T} & B_{x 2}^{T} & B_{y 2}^{T} & B_{x 5}^{T} & B_{y 5}^{T} & B_{x 4}^{T} & B_{y 4}^{T} & B_{x 3}^{T} & B_{y 3}^{T} & 0
\end{array}\right)\left(\begin{array}{l}
u_{x 1} \\
u_{y 1} \\
u_{x 2} \\
u_{y 2} \\
u_{x 5} \\
u_{y 5} \\
u_{x 4} \\
u_{y 4} \\
u_{x 3} \\
u_{y 3} \\
p_{4}
\end{array}\right)\left(\begin{array}{c}
f x_{1} \\
f y_{1} \\
f x_{2} \\
f y_{2} \\
f x_{5} \\
f y_{5} \\
f x_{4} \\
f y_{4} \\
f x_{3} \\
f y_{3} \\
0
\end{array}\right) .
$$

In this model, the coefficient matrix $\mathrm{A}$ has an $11 \times 11$ block structure, with 10 sets of velocity variables and 1 set of pressure variables, $p_{4}$.

Our new preconditioning $\mathrm{P}$ can be extended to include the extra phase variables as follows:

$$
P_{1}=\left(\begin{array}{cccccccccccc}
k_{x x 11} & k_{x y 11} & k_{x x 12} & 0 & k_{x x 15} & 0 & k_{x x 14} & 0 & k_{x x 13} & 0 & C_{x 1} \\
0 & k_{y y 11} & 0 & k_{y y 12} & 0 & k_{y y 15} & 0 & k_{y y 14} & 0 & k_{y y 13} & C_{y 1} \\
0 & 0 & k_{x x 22} & k_{x y 22} & k_{x x 25} & 0 & k_{x x 24} & 0 & k_{x x 23} & 0 & C_{x 2} \\
0 & 0 & 0 & k_{y y 22} & 0 & k_{y y 25} & 0 & k_{y y 24} & 0 & k_{y y 23} & C_{y 2} \\
0 & 0 & 0 & 0 & k_{x x 55} & k_{x y 55} & k_{x x 54} & 0 & k_{x x 53} & 0 & C_{x 5} \\
0 & 0 & 0 & 0 & 0 & k_{y y 55} & 0 & k_{y y 54} & 0 & k_{y y 53} & C_{y 5} \\
0 & 0 & 0 & 0 & 0 & 0 & k_{x x 44} & k_{x y 44} & k_{x x 43} & 0 & C_{x 4} \\
0 & 0 & 0 & 0 & 0 & 0 & 0 & k_{y y 44} & 0 & k_{y y 43} & C_{y 4} \\
0 & 0 & 0 & 0 & 0 & 0 & 0 & 0 & k_{x x 33} & k_{x y 33} & 0 \\
0 & 0 & 0 & 0 & 0 & 0 & 0 & 0 & 0 & k_{y y 33} & 0 \\
0 & 0 & 0 & 0 & 0 & 0 & 0 & 0 & 0 & 0 & m p
\end{array}\right) .
$$


As before, we approximate the inverse of each diagonal block by a single AMG V-cycle, except for the pressure mass matrix which approximated by its diagonal. The performance of our preconditioner for the five-phase model is just as effective as for the four-phase model. Table 5 presents the running time and the GMRES iterations that are required to solve the linear momentum system (19) on a sequence of grids. The computational time again increased by factor of nearly 4 as $\mathrm{N}$ is quadrupled, which means that the solver requires approximately $\mathcal{O}(N)$ complexity. Moreover, The GMRES iterations in this case are again nearly independent on the grid size. The cost of the whole new model solution on fully unstructured grids is also reported in Table 5. It can be observed that the CPU time and the memory requirement for the whole solvers scale approximately linearly and so behave as $\mathcal{O}(N)$.

Table 5: The average running times (in seconds), over 1000 time steps, required for solving the linear algebraic system (19) using $P_{1}$ preconditioned GMRES on fully unstructured grids and the CPU time and percentage of memory cost for the whole model on unstructured grids: GI is GMRES iterations, $\mathrm{N}$ is the number of unknowns.

\begin{tabular}{c|cc|cc} 
& \multicolumn{2}{|c|}{ Solution of the momentum equation } & \multicolumn{2}{c}{ Solution of the whole model } \\
\hline $\mathrm{N}$ & Average GI & Average time per step & MEM $(\%)$ & Time \\
\hline 94709 & 41.9560 & 2.7443692667 & 3.2 & $50 \mathrm{~m} 58 \mathrm{~s}$ \\
375506 & 44.5614 & 11.91469505 & 10.3 & $220 \mathrm{~m} 35 \mathrm{~s}$ \\
1495397 & 42.1758 & 46.629512359 & 34.4 & $867 \mathrm{~m} 25 \mathrm{~s}$ \\
\hline
\end{tabular}

\section{Conclusion}

In this paper we have studied the multiphase model of vascular tumour growth in two-dimensions, which is presented by Hubbard and Byrne [1]. Efficient computational algorithms have been developed for the numerical approximation of these PDE systems describing multiphase flow. In particular we improved the efficiency of the numerical methods used in [1] in order to reach optimal efficiency.

We believe that this work opens a number of possibilities for future research in this area. There are two main avenues. Firstly we could exploit this optimal efficiency across a broader range of multiphase flow problems. This could include: 
- Using different numbers of phases in tumour model: our preconditioner makes it possible to model multiphase problem with many more phases.

- Modelling different drug performance, for example attempting to use the pharmacology of a drug such as in [24], taking sufficient fine spatial meshes to ensure mesh convergence.

- Using multiphase incompressible flow models in completely different applications, such as [25] and [26] for example.

Secondly, it is possible extend the existing model to 3D, still using our efficient algorithms. The important issues are extending the computational algorithms to 3D problems, and making them efficient enough to be practical for real computations. In three-dimensions, for the four-phase model, the mass balance equation and the reaction-diffusion equation could be extended to 3D using tetrahedral meshes. The momentum balance equations will need to include three velocities for each phase, $(\mathrm{u}, \mathrm{v}, \mathrm{w})$, and one pressure variable. Moreover the same discretization schemes of these PDEs can be used in 3D (i.e quadratic velocities and linear pressures). Our preconditioner in $3 \mathrm{D}$ would then be:

$$
P=\left(\begin{array}{cccccccccccccc}
k_{x x 11} & k_{x y 11} & k_{x z 11} & k_{x x 12} & 0 & 0 & k_{x x 14} & 0 & 0 & k_{x x 13} & 0 & 0 & C_{x 1} \\
0 & k_{y y 11} & k_{y z 12} & 0 & k_{y y 12} & 0 & 0 & k_{y y 14} & 0 & 0 & k_{y y 13} & 0 & C_{y 1} \\
0 & 0 & k_{z z 11} & 0 & 0 & k_{z z 12} & 0 & 0 & k_{z z 14} & 0 & 0 & k_{z z 13} & C_{z 1} \\
0 & 0 & 0 & k_{x x 22} & k_{x y 22} & k_{x z 22} & k_{x x 24} & 0 & 0 & k_{x x 23} & 0 & 0 & C_{x 2} \\
0 & 0 & 0 & 0 & k_{y y 22} & k_{y z 22} & 0 & k_{y y 24} & 0 & 0 & k_{y y 23} & 0 & C_{y 2} \\
0 & 0 & 0 & 0 & 0 & k_{z z 22} & 0 & 0 & k_{z z 24} & 0 & 0 & k_{z z 23} & C_{z 2} \\
0 & 0 & 0 & 0 & 0 & 0 & k_{x x 44} & k_{x y 44} & k_{x z 44} & k_{x x 43} & 0 & 0 & C_{x 4} \\
0 & 0 & 0 & 0 & 0 & 0 & 0 & k_{y y 44} & k_{y z 44} & 0 & k_{y y 43} & 0 & C_{y 4} \\
0 & 0 & 0 & 0 & 0 & 0 & 0 & 0 & k_{z z 44} & 0 & 0 & k_{z z 43} & C_{z 4} \\
0 & 0 & 0 & 0 & 0 & 0 & 0 & 0 & 0 & k_{x x 33} & k_{x y 33} & k_{x z 33} & 0 \\
0 & 0 & 0 & 0 & 0 & 0 & 0 & 0 & 0 & 0 & k_{y y 33} & k_{y z 33} & 0 \\
0 & 0 & 0 & 0 & 0 & 0 & 0 & 0 & 0 & 0 & 0 & k_{z z 33} & 0 \\
0 & 0 & 0 & 0 & 0 & 0 & 0 & 0 & 0 & 0 & 0 & 0 & m p
\end{array}\right) .
$$

\section{Acknowledgements}

Ahlam Hamdan Alrehaili is supported by a PhD scholarship from Taibah University, Medina, Saudi Arabia.

\section{References}

[1] M. E. Hubbard, H. M. Byrne, Multiphase modelling of vascular tumour growth in two spatial dimensions, Journal of Theoretical Biology 316 (16) (2013) 70-89. doi:10.1016/j.jtbi.2012.09.031. 
[2] H. M. Byrne, T. Alarcon, M. R. Owen, S. D. Webb, P. K. Maini, Modelling aspects of cancer dynamics: a review, Philosophical Transactions of the Royal Society of London A: Mathematical, Physical and Engineering Sciences 364 (1843) (2006) 1563-1578. doi:10.1098/rsta.2006.1786.

[3] C. J. W. Breward, H. M. Byrne, C. E. Lewis, A multiphase model describing vascular tumour growth, Bulletin of Mathematical Biology 65 (4) (2003) 609-640. doi:10.1016/S0092-8240(03)00027-2.

[4] C. J. W. Breward, H. M. Byrne, C. E. Lewis, The role of cell-cell interactions in a two-phase model for avascular tumour growth, Journal of Mathematical Biology 45 (2) (2002) 125-152. doi:10.1007/s002850200149.

[5] J. P. Ward, J. R. King, Mathematical modelling of avasculartumour growth II: modelling growth saturation, Mathematical Medicine and Biology: A Journal of the IMA 16 (2) (1999) 171-211. doi:10.1093/imammb/16.2.171.

[6] T. Roose, S. J. Chapman, P. K. Maini, Mathematical models of avascular tumour growth, SIAM Review 49 (2) (2007) 179-208. doi:10.1137/S0036144504446291.

[7] M. E. Orme, M. A. J. Chaplain, A mathematical model of vascular tumour growth and invasion, Mathematical and Computer Modelling 23 (10) (1996) 43-60. doi:10.1016/0895-7177(96)00053-2.

[8] G. Sciumè, S. Shelton, W. G. Gray, C. T. Miller, F. Hussain, M. Ferrari, P. Decuzzi, B. A. Schrefler, A multiphase model for threedimensional tumour growth, New Journal of Physics 15 (1) (2013) 015005. doi:10.1088/1367-2630/15/1/015005.

[9] C. Y. Wang, P. Cheng, A multiphase mixture model for multiphase, multicomponent transport in capillary porous media-I. model development, International Journal of Heat and Mass Transfer 39 (17) (1996) 3607-3618. doi:10.1016/0017-9310(96)00036-1.

[10] C. E. Brennen, Fundamentals of multiphase flow, Cambridge University Press, 2005. 
[11] H. C. Elman, D. J. Silvester, A. J. Wathen, Finite Elements and Fast Iterative Solvers, Oxford University Press, 2005.

[12] A. Wathen, D. Silvester, Fast iterative solution of stabilised stokes systems. part 1: using simple diagonal preconditioners, SIAM Journal on Numerical Analysis 30 (3) (1993) 630-649.

[13] A. Wathen, D. Silvester, Fast iterative solution of stabilised stokes systems. part 2: using general block preconditioners, SIAM Journal on Numerical Analysis 31 (5) (1994) 1352-1367.

[14] D. Kay, D. Loghin, A. Wathen, A preconditioner for the steadystate navier-stokes equations, SIAM Journal on Scientific Computing 21 (1) (2002) 237-256.

[15] H. C. Elman, D. Loghin, A. J. Wathen, Preconditioning techniques for newton's method for the incompressible navier-stokes equations, BIT 43 (5) (2003) 961-974.

[16] M. F. Murphy, G. H. Golub, A. J. Wathen, A note on preconditioning for indefinite linear systems, SIAM Journal on Scientific Computing 21 (6) (2000) 1969-1972.

[17] R. J. LeVeque, Finite volume methods for hyperbolic problems, Vol. 31, Cambridge University Press, 2002.

[18] A. H. Alrehaili, Efficient iterative solution algorithms for numerical models of multiphase flow, Ph.D. thesis, School of Computing, University of Leeds (2018).

URL http://etheses.whiterose.ac.uk/20292

[19] MUMPS, A multifrontal massively parallel sparse direct solver. URL http://graal.ens-lyon.fr/MUMPS/

[20] A. J. Wathen, Preconditioning and fast solvers for incompressible flow, Tech. rep., Oxford University Computing Laboratory (2004). URL http://eprints.maths.ox.ac.uk/1182/

[21] A. J. Wathen, Preconditioning, Acta Numerica 24 (2015) 329-376. 
[22] HSL, A collection of Fortran codes for large-scale scientific computation.

URL http://www.hsl.rl.ac.uk

[23] J. Boyle, M. D. Mihajlovic, J. A. Scott, HSL MI20: an efficient AMG preconditioner, Tech. rep., RAL-TR-2007-021, STFC Rutherford Appleton Laboratory, Didcot, UK, (2007).

[24] C. M. Groh, M. E. Hubbard, P. F. Jones, P. M. Loadman, N. Periasamy, B. D. Sleeman, S. W. Smye, C. J. Twelves, R. M. Phillips, Mathematical and computational models of drug transport in tumours, Journal of The Royal Society Interface 11 (94) (2014) 20131173. doi:10.1098/rsif.2013.1173.

[25] L. Baňas, R. Nürnberg, Numerical approximation of a non-smooth phase-field model for multicomponent incompressible flow, ESAIM: Mathematical Modelling and Numerical Analysis 51 (3) (2017) 1089-1117. doi:10.1051/m2an/2016048.

[26] G. B. Wright, R. D. Guy, A. L. Fogelson, An efficient and robust method for simulating two-phase gel dynamics, SIAM Journal on Scientific Computing 30 (5) (2008) 2535-2565. doi:10.1137/070695927. 